\title{
Angiography-negative childhood primary angiitis of the central nervous system diagnosed by open brain biopsy: a case report
}

\author{
Dayun Kang ${ }^{1}$, Soo Yeon Kim ${ }^{1}$, Jong Hee Chae ${ }^{1}$, Ki Joong Kim ${ }^{1}$, Sung-Hye Park², Byung Chan Lim ${ }^{1}$ \\ ${ }^{1}$ Department of Pediatrics, Seoul National University Children's Hospital, Seoul National University College of Medicine, Seoul, Korea \\ ${ }^{2}$ Department of Pathology, Seoul National University Hospital, Seoul National University College of Medicine, Seoul, Korea
}

Childhood primary angiitis of the central nervous system (PACNS) is rare and has been poorly defined, which makes it difficult to diagnose and treat. Herein, we report a case of childhood PACNS that was diagnosed by open brain biopsy. Clinical symptoms and radiologic findings improved after combination treatment with steroid and cyclophosphamide. In this case, a 16-year-old, previously healthy, adolescent male complained of headache, seizure, and right-side weakness with hypoesthesia. Brain magnetic resonance imaging (MRI) showed multifocal, high-signal intensity lesions on T2-weighted scans with patch contrast enhancement. The clinical symptoms improved after intravenous steroid pulse therapy (methylprednisolone, 1,000 mg/day for 3 consecutive days) and subsequent oral steroid maintenance. However, follow-up brain MRI showed aggravation of the previous lesions. Open brain biopsy of the left parietal lobe showed infiltration of lymphoplasma cells to the vessel walls with parenchymal necrosis, consistent with PACNS. The patient received four monthly intravenous cyclophosphamide (1,000 mg/dose at each cycle) treatments along with oral steroid maintenance. After treatment, he was symptom-free, and follow-up MRI revealed marked lesion improvements. This case suggests the important role of brain biopsy and aggressive immunosuppressive treatment in diagnosis and management of childhood PACNS.

Keywords: Primary angiitis of the central nervous system, Cyclophosphamide, Immunosuppressive treatment, Open brain biopsy

\section{Introduction}

Primary angiitis of the central nervous system (PACNS) in children is a rare and poorly understood disease that can cause severe neurological problems. Children with PACNS present with various neurological symptoms, from mild (e.g., headache, fever, fatigue, concentration difficulties) to severe (e.g., intractable seizures, hemiparesis, hemisensory loss, cognitive dysfunction, mood and/or personality changes, decreased consciousness) [1,2]. Various neurological outcomes have been reported; some children with PACNS have undergone devastating courses that progressed to death, while others have shown complete neurological recovery after appropriate immunosuppressive treatment $[3,4]$. These outcomes suggest that the neurological deficits caused by brain inflam- mation can be reversible, and that appropriate immunosuppressive treatment is necessary to cure the disease.

In adults, Calabrese and Mallek [5] proposed a set of diagnostic criteria for PACNS, including (i) a newly acquired neurologic deficit, (ii) angiographic and/or histologic features of central nervous system (CNS) vasculitis, and (iii) no evidence of a systemic condition associated with the findings. However, diagnostic criteria for childhood PACNS have not been established, and definitive diagnosis requires both confirmation by pathology and exclusion of disorders that mimic the disease.

Children with PACNS can be classified into two groups based on conventional angiography results; angiography positive and angiography negative. Angiography-positive PACNS usu-

Received: September 11, 2021 Revised: October 28, 2021 Accepted: November 1, 2021

Correspondence: Byung Chan Lim

Department of Pediatrics, Seoul National University Children's Hospital, 101 Daehak-ro, Jongno-gu, Seoul 03080, Korea

E-mail: prabbit7@snu.ac.kr

ORCID: https://orcid.org/0000-0002-8509-4135

Copyright (C) 2022 by The Korean Encephalitis and Neuroinflammation Society

This is an open access article distributed under the terms of the Creative Commons Attribution Non-Commercial License (http://creativecommons.org/licenses/by-nc/4.0/) which permits unrestricted non-commercial use, distribution, and reproduction in any medium, provided the original work is properly cited. 
ally causes inflammation in medium to large vessels, while angiography-negative PACNS usually is associated with small vessels. Although angiography-negative PACNS is associated with more severe clinical features such as seizure, cognitive dysfunction, or speech changes and needs more aggressive treatment compared with angiography-positive PACNS, information about diagnosis and treatment of angiography-negative PACNS is limited [6,7]. As angiography-negative PACNS often is underevaluated due to its nonspecific clinical presentation and laboratory and imaging findings, brain biopsy often is needed to confirm this diagnosis.

In previous case reports and case series, treatments for angiography-negative PACNS have included intravenous or oral corticosteroids, cyclophosphamide, azathioprine, mycophenolate mofetil, methotrexate, intravenous gamma globulin, and infliximab at varying doses and for different durations [812]. Only one open-label cohort study by Hutchinson et al. [2] proposed a treatment protocol of 6 months of induction therapy with oral prednisone and intravenous cyclophosphamide, followed by 18 months of maintenance therapy with oral mycophenolate mofetil or azathioprine.

Herein, we report a case of a 16-year-old boy with angiography-negative childhood PACNS, which was confirmed by open brain biopsy, who achieved good 1-year follow-up recovery outcomes after combined corticosteroid and cyclophosphamide therapy. This case demonstrates the importance of tissue biopsy in cases of suspected PACNS in children. The preferred treatment is combined corticosteroid and cyclophosphamide therapy, which was associated with successful results in this case.

\section{Case Report}

A 16-year-old boy presented with generalized tonic-clonic seizure that lasted for 2 minutes. Projectile vomiting and right-side motor weakness with hypoesthesia followed the seizure event. The patient had a 3-year history of severe headaches, though brain magnetic resonance imaging (MRI) 1 year prior to the event showed no abnormality. Fever and myalgia with upper respiratory symptoms occurred 2 weeks before the seizure. The patient's height was $179 \mathrm{~cm}$ (90th percentile), and weight was $106.3 \mathrm{~kg}$ ( $>97$ th percentile). Brain MRI revealed T2 high-signal intensity lesions in the left parietal, right frontal, and right temporal areas, associated with patch contrast enhancement (Figure 1A-D). Whole blood white blood cell (WBC) count was elevated $\left(10,420 / \mathrm{mm}^{3}\right)$. Se- rum C-reactive protein (CRP) and erythrocyte sedimentation rate (ESR) were increased; CRP, $0.99 \mathrm{mg} / \mathrm{dL}$ (reference range, 0-0.5 mg/dL) and ESR, $31 \mathrm{~mm} / \mathrm{hr}$ (reference range, 0-9 mm/ hr). Cerebrospinal fluid (CSF) examination showed normal WBC count and protein level; $\mathrm{WBC}, 4 / \mathrm{mm}^{3}$ and protein, 35 $\mathrm{mg} / \mathrm{dL}$. CSF cytospin showed no evidence of malignancy. Serum antinuclear antibody, antineutrophil cytoplasmic antibody, rheumatoid factor, and antiphospholipid antibodies were all negative. Complements 3 and 4 were within normal limits. Anti-aquaporin 4 antibody and antimyelin oligodendrocyte glycoprotein antibody results were negative. CSF viral polymerase chain reaction studies including Epstein-Barr virus, herpes simplex 1 and 2 viruses, and human herpesvirus 6 were negative. Serum immunoglobulin G antibodies to Taenia solium and Sparganums were negative. Because we were under the impression that the patient had an acute demyelinating syndrome, such as acute disseminated encephalomyelitis (ADEM) or autoimmune encephalitis, he was treated with steroid pulse therapy (methylprednisolone, 1,000 mg/day for 3 consecutive days) and subsequent oral steroid maintenance. After steroid treatment, his headache was improved, and there were no additional seizure events. However, 1-month follow-up MRI after steroid pulse therapy showed aggravation of the lesions (Figure 1E-H). Open brain biopsy of the left parietal lobe was performed, and the results showed infiltration of lymphoplasma cells, histiocytes, T-cells, and B-cells around the small blood vessel walls. Parenchymal necrosis (granuloma) of the brain was observed along with inflammation, consistent with the vasculitis noted on histopathology (Figure 2).

Transfemoral cerebral angiography showed no steno-occlusive lesions or abnormal collateral vessels on cerebral and vertebral arteries. The patient received four monthly intravenous cyclophosphamide $(1,000 \mathrm{mg} /$ dose at each cycle) treatments along with oral steroid maintenance. He remained symptom-free, and brain MRI following the two initial cycles of cyclophosphamide and the four subsequent cycles of cyclophosphamide revealed marked improvement of the lesions (Figure 1I-L and 1M-P, respectively). Six months after completing the final four cycles of cyclophosphamide, no recurred lesions were observed (Figure 1Q-T).

This study was performed under the principles of the Declaration of Helsinki, and informed consent was obtained from the patient and his parents. 
Figure 1 Serial brain MRI findings at onset (A-D), 1 month after onset, after steroid treatment (E-H), 3 months after onset, after two cycles of intravenous cyclophosphamide (I-L), after additional two cycles (total four cycles) of intravenous cyclophosphamide $(\mathrm{M}-\mathrm{P})$, and 1 year after onset $(\mathrm{Q}-\mathrm{T})$

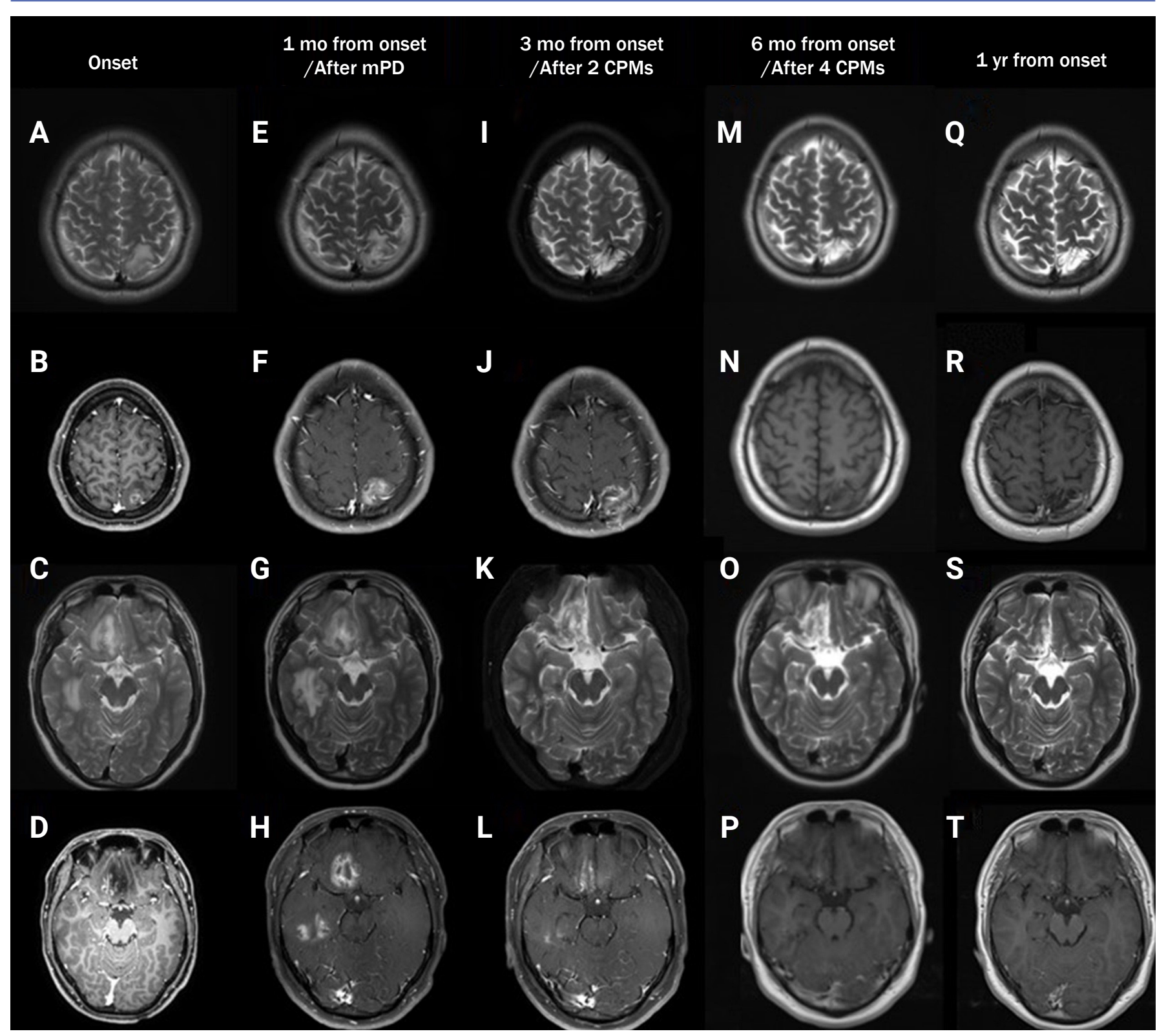

The area of T2 high-signal intensity and contrast enhancement increased at 1 month after onset of steroid treatment compared with that at onset. After a combined treatment approach with intravenous cyclophosphamide (CPM) and oral steroids, the area of the T2 high-signal intensity and contrast enhancement dramatically decreased.

$\mathrm{mPD}$, methylprednisolone pulse therapy.

\section{Discussion}

The patient initially presented with symptoms and brain MRI findings that were not specific to PACNS. Steroid pulse and maintenance treatment was initiated based on initial differential diagnosis of acute demyelinating syndrome, such as
ADEM. Although the clinical symptoms improved quickly after this treatment, follow-up brain MRI revealed aggravation of the lesions, which is unusual in ADEM. Alternate diagnoses that should have been considered include vasculitis, CNS malignancy, and rare causes of infection. We decided to perform open brain biopsy because extensive evaluations did not re- 


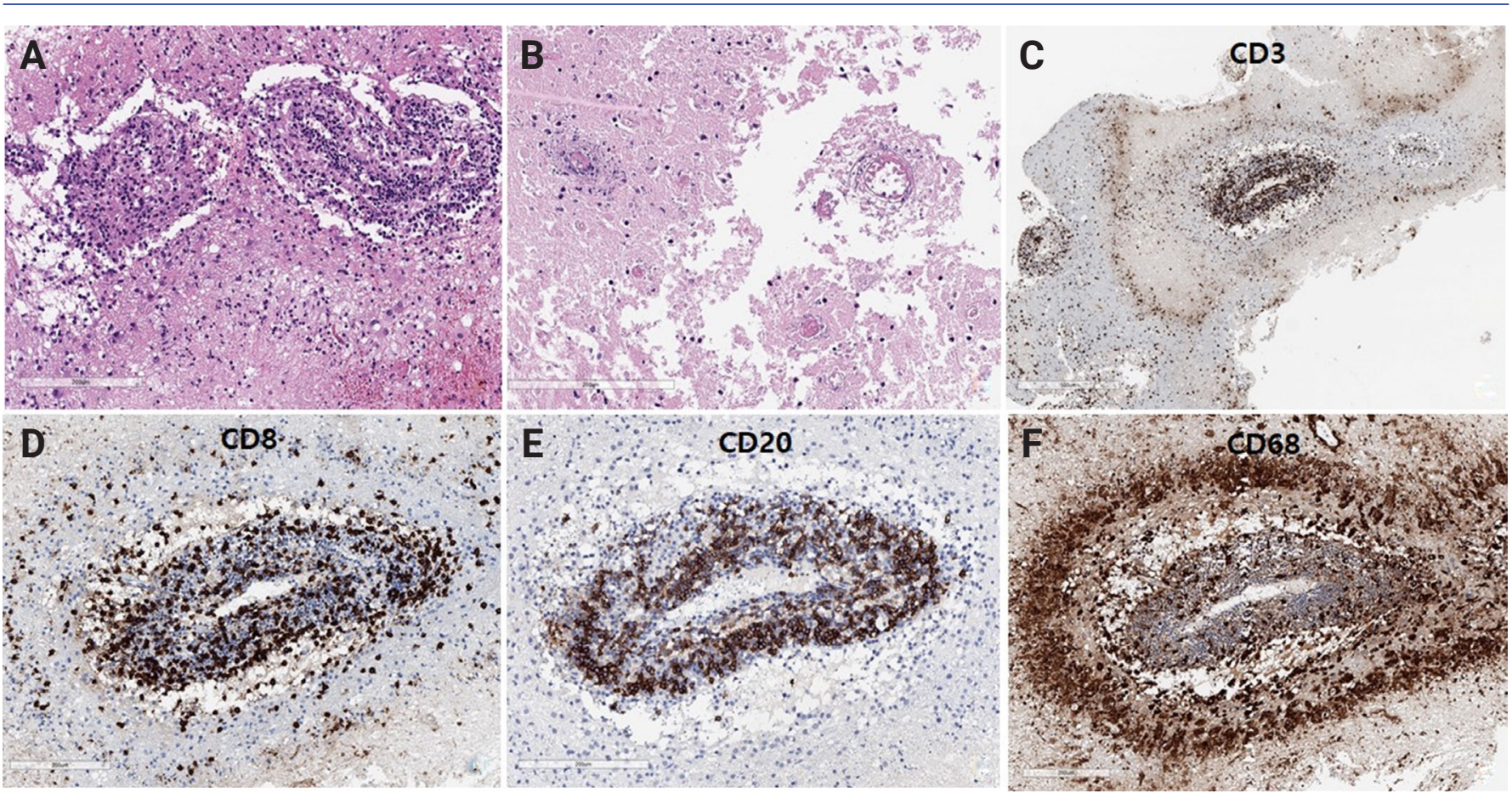

(A) The small blood vessels and leptomeningeal blood vessels showed lymphoplasma cellular infiltration in the vessel wall. (B) The brain parenchyma showed a large area of infarction with a trace of blood vessels. (C) CD3 immunostain revealed positive T-cells in the blood vessel and perivascular area. (D, E, F) Imaging revealed that the blood vessel was infiltrated by CD8-positive T-cells, CD20-positive B-cells, and CD68-positive histiocytes. The B-cells were mainly located in the blood vessel, but CD8-T-cells also were found in the brain parenchyma. The histiocytic cells were densely infiltrated around the blood vessel.

A, B: H\&E stain; C: CD3 immunostain; D: CD8 immunostain; E: CD20 immunostain; F: CD68 immunostain. Scale bars in A-F, $200 \mu m$.

veal any diagnostic indications. We confirmed the PACNS diagnosis and added cyclic intravenous cyclophosphamide treatment. This case highlights the importance of brain biopsy for confirming a diagnosis and guiding appropriate treatment in pediatric patients with undetermined inflammatory CNS diseases. One important consideration for patients is that brain biopsy can have limited sensitivity (50\%-75\%) due to the uneven distribution of vasculitis lesions [13,14]. To overcome this limitation, sampling both the leptomeninges and underlying cortex is recommended to increase the diagnostic yield $[15,16]$. Similarly, biopsy of a radiographically abnormal area, particularly in the presence of abnormal enhancement, could improve the sensitivity of the procedure [16].

Many pediatric case reports have described good responses to combined steroids plus oral or intravenous cyclophosphamide therapy $[2,6,11]$. The reported duration and intervals for drug administration in the reports have varied and were determined mainly based on each patient's symptomatic and radiological improvement. Hutchinson et al. [2] recruited 19 pediatric patients with small-vessel PACNS and used a uniform treatment protocol of 6 months of induction and 18 months of maintenance therapy. They administered seven pulses of 500 to $750 \mathrm{mg} / \mathrm{m}^{2}$ intravenous cyclophosphamide every 4 weeks, and prolonged oral prednisolone was administered during induction therapy. Mycophenolate mofetil or azathioprine was maintained during the maintenance period. The authors concluded that this aggressive, prolonged immune suppression could contribute to relatively favorable clinical outcomes. Therefore, we administered four cycles of intravenous cyclophosphamide treatment $(1,000 \mathrm{mg}$ at each cycle) and maintained 6 months of oral prednisolone for the patient in this report. We reduced the number of intravenous cyclophosphamide cycles and decided against maintenance therapy because the clinical symptoms improved quickly after steroid treatment and brain MRI lesions revealed marked improvement after two cycles of intravenous cyclophosphamide. Although the follow-up duration after therapy was insufficient to completely evaluate our treatment regimen, there was no 
clinical or radiologic evidence of relapse after the 12-month follow-up. Considering the relatively high toxicity profile of immunosuppressive treatment and limited data on the natural history of childhood PACNS, the immunosuppressive treatment duration must be individualized depending on clinical and radiologic treatment responses. We suggest using regular brain MRI imaging follow-up to predict disease activity and detect early disease flare-up. If imaging changes are observed, additional treatment should be considered. Although inflammation markers such as CRP or ESR do not always accompany disease flare, the results might help identify early stages of disease flare-up [6].

\section{CONCLUSION}

We present the case of a 16-year-old male adolescent with childhood small-vessel PACNS, confirmed by open brain biopsy and treated with combined corticosteroid and cyclophosphamide. Confirmation of PACNS by brain biopsy in the early disease course can lead to earlier administration of appropriate immunosuppressive treatment and prevent longterm neurological deficits. This approach can be beneficial for patients and should be considered during diagnostic differentiation by clinicians.

\section{Conflicts of Interest}

No potential conflict of interest relevant to this article was reported.

\section{Author Contributions}

Conceptualization: Lim BC; Data curation, Project administration: Kang D; Resources: Park SH; Supervision: Kim SY, Chae JH, Kim KJ; Writing-original draft: Kang D; Writing-review \& editing: all authors.

\section{References}

1. Benseler SM, Silverman E, Aviv RI, et al. Primary central nervous system vasculitis in children. Arthritis Rheum 2006;54:1291-7.

2. Hutchinson C, Elbers J, Halliday W, et al. Treatment of small vessel pri- mary CNS vasculitis in children: an open-label cohort study. Lancet Neurol 2010;9:1078-1084.

3. Gallagher KT, Shaham B, Reiff A, et al. Primary angiitis of the central nervous system in children: 5 cases. J Rheumatol 2001;28:616-623.

4. Matsell DG, Keene DL, Jimenez C, Humphreys P. Isolated angiitis of the central nervous system in childhood. Can J Neurol Sci 1990; 17:151-154.

5. Calabrese LH, Mallek JA. Primary angiitis of the central nervous system: report of 8 new cases, review of the literature, and proposal for diagnostic criteria. Medicine (Baltimore) 1988;67:20-39.

6. Cellucci T, Tyrrell PN, Sheikh S, Benseler SM. Childhood primary angiitis of the central nervous system: identifying disease trajectories and early risk factors for persistently higher disease activity. Arthritis Rheum 2012;64:1665-1672.

7. Beelen J, Benseler SM, Dropol A, Ghali B, Twilt M. Strategies for treatment of childhood primary angiitis of the central nervous system. Neurol Neuroimmunol Neuroinflamm 2019;6:e567.

8. Nishida H, Kumada S, Komori T, et al. IVIG in childhood primary angiitis of the central nervous system: a case report. Brain Dev 2020;42:675-679.

9. Batthish M, Banwell B, Laughlin S, et al. Refractory primary central nervous system vasculitis of childhood: successful treatment with infliximab. J Rheumatol 2012;39:2227-2229.

10. Yaari R, Anselm IA, Szer IS, Malicki DM, Nespeca MP, Gleeson JG. Childhood primary angiitis of the central nervous system: two biopsy-proven cases. J Pediatr 2004;145:693-697.

11. Lanthier S, Lortie A, Michaud J, Laxer R, Jay V, deVeber G. Isolated angiitis of the CNS in children. Neurology 2001;56:837-842.

12. Benseler SM, deVeber G, Hawkins C, et al. Angiography-negative primary central nervous system vasculitis in children: a newly recognized inflammatory central nervous system disease. Arthritis Rheum 2005;52:2159-2167.

13. Calabrese LH, Duna GF, Lie JT. Vasculitis in the central nervous system. Arthritis Rheum 1997;40:1189-1201.

14. Duna GF, Calabrese LH. Limitations of invasive modalities in the diagnosis of primary angiitis of the central nervous system. J Rheumatol 1995;22:662-667.

15. Calabrese LH, Furlan AJ, Gragg LA, Ropos TJ. Primary angiitis of the central nervous system: diagnostic criteria and clinical approach. Cleve Clin J Med 1992;59:293-306.

16. Parisi JE, Moore PM. The role of biopsy in vasculitis of the central nervous system. Semin Neurol 1994;14:341-348. 